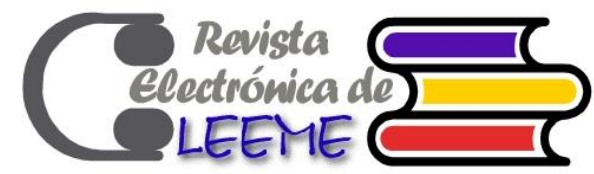

Electronic Journal of Music in Education

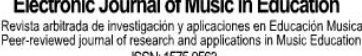

ISSN: 15759563

\title{
La recreación artística de textos e imágenes como modelo para el desarrollo de la creatividad y la integración de los lenguajes expresivos en el aula de Música
}

Texts and images artistic recreation as a model for the development of the creativity and the integration of the expressive languages in the music classroom

Juan Jesús Yelo Cano jjyelo@um.es

Facultad de Educación

Universidad de Murcia

Murcia, España

ORCID: http://orcid.org/0000-0001-8081-9523

doi: 10.7203/LEEME.42.13171

Recibido: 15-10-2018 Aceptado: 00-12-2018. Contacto y correspondencia: Juan Jesús Yelo Cano, Departamento de Expresión Plástica, Musical y

Dinámica

Universidad de Murcia, Campus de Espinardo, 30100 Murcia. España.

\section{Resumen}

La finalidad de este artículo es presentar la experiencia realizada en un Instituto de Educación Secundaria de la Región de Murcia durante el curso 2017-2018 en la asignatura "Artes Escénicas y Danza". En la primera fase de este trabajo, alumnas de $4^{\circ}$ de Educación Secundaria Obligatoria iniciaron una serie de procesos de reflexión, acción y valoración para recrear algunos de los textos y fotografías presentes en la exposición "Paisajes con alma". Las actividades realizadas desembocaron en la creación de una obra en un lenguaje estético diferente al original en la que se integren danza, expresión corporal, videoarte, arte sonoro, música concreta, etc. En una segunda fase de difusión (aún por realizar), las elaboraciones artísticas resultantes serán mostradas al público acompañando a la muestra en forma de códigos QR. La motivación de los participantes por la tarea expresiva a realizar, las elaboraciones artísticas resultantes y la satisfacción por el trabajo efectuado demuestran el éxito educativo de la experiencia.

Palabras clave: Creatividad, integración de las artes, educación musical, arte contemporáneo.

Abstract

The purpose of this article is to present the experience carried out at a Secondary School in the Region of Murcia during the 20172018 school year in the "Performing Arts and Dance" subject. In the first phase of this work, students of 4th year of Secondary Education initiated a series of reflection, action and assessment processes to recreate some of the texts and photographs present in the exhibition "Paisajes con alma". The activities carried out unleash the creation of a work in an aesthetic language different from the original that integrate dance, body language, video art, sound art, concrete music, etc. In a second phase of dissemination (still to be carried out), the resulting artistic elaborations are shown to the public accompanying the exhibition in the form of QR codes. The motivation of the participants and the satisfaction for the work done demonstrate the success of the experience.

Key words: Creativity, integrated arts, music education, contemporary art. 


\section{Introducción}

Las artes son una actividad universal que define a los grupos humanos. A lo largo de la historia, las experiencias estéticas han contribuido al crecimiento intelectual y al desarrollo emocional de las comunidades. Por otra parte, la creatividad es una cualidad cada vez más apreciada en nuestra sociedad hasta el extremo de ser considerada uno de los valores con más proyección. La capacidad para idear y llevar a la práctica proyectos complejos incluye la puesta en marcha de muy variados procesos cognitivos. Es cierto que esto no es algo exclusivo del arte, pero es en el campo artístico donde los citados mecanismos parecen ser más evidentes para el público en general.

Dentro de las últimas tendencias en educación, existen muchas experiencias que nos muestran cómo la integración de las artes en los centros escolares es necesaria por su valor intrínseco como medio para desarrollar las capacidades del alumnado. El acto creador se manifiesta como algo que satisface a su autor y genera la confianza necesaria para avanzar de forma optimista en el logro del éxito. La comprensión de los procesos artísticos posee la facultad de otorgar al alumnado una vía de conocimiento y aprehensión de la realidad diferente y complementaria a la que puede proporcionarle la ciencia.

\section{La integración de los lenguajes artísticos}

La multidisciplinariedad en el arte contemporáneo es algo indiscutible. La diferenciación impuesta en el siglo XVIII entre artes espaciales y temporales acaba con la transversalidad del sonido en el arte, sobre todo, con las relaciones entre arquitectura y sonido. Pero a principios de siglo XX y contando como precedente la idea romántica de "obra de arte total", las fronteras entre las disciplinas artísticas se van desdibujando. Takkal y Rubio nos recuerdan que Adorno describe en 1966 en El Arte y las artes cómo se van produciendo "imbricaciones, yuxtaposiciones y fusiones entre las diversas artes que de acuerdo con sus propias leyes de movimiento se resistían a permanecer en sus zonas" (Takkal y Rubio, 2017, pp. 28-29).

La propia concepción de arte sonoro es un claro ejemplo de cómo se han ido diluyendo las fronteras entre las artes. Molina (2017) indica que:

"La práctica suele anteceder a su conceptualización y el arte sonoro podemos considerarlo que ha estado allí presente en toda la historia donde no estaba su nombre o su nombre era otro. (...) hemos encontrado (...) indicios de algunos aspectos de la práctica del arte sonoro: poesía fonética, escultura sonora, radio-arte, paisaje sonoro, acciones sonoras, entre otras" (pp. 17-19).

En un sentido estricto, el término arte sonoro comienza a utilizarse a finales de los años setenta del siglo pasado en relación con ciertos trabajos audiovisuales que utilizaban el sonido en un sentido amplio que no estaba sometido a las restricciones de la música académica. Sin embargo, Rudi (2009) advierte que el arte sonoro "es un término difuso, no bien definido y que absorbe nuevas prácticas artísticas tan rápidamente que corre el peligro de fracasar como una JUAN JESÚS YELO CANO. THE CONTENT OF THIS ARTICLE IS THE SOLE RESPONSIBILITY OF THE AUTHORS. THE REVISTA ELECTRÓNICA DE LEEME AND UNIVERSITAT DE VALĖNCIA ARE NOT LIABLE FOR ANY LEGAL ACTIONS THAT MAY ARISE INVOLVING THE ARTICLE'S CONTENT. REVISTA ELECTRÓNICA DE LEEME -LISTA ELECTRÓNICA EUROPEA DE MÚSICA EN LA EDUCACIÓN-. HTTP://OJS.UV.ES/INDEX.PHP/LEEME/INDEX ISSN: 1575-9563 EDITORES: UNIVERSIDAD DE VALENCIA Y JESÚS TEJADA GIMÉNEZ. VISIBILIDAD DE ESTA REVISTA: SCOPUS, EMERGING CITATION INDEX (CLARIVATE), EBSCO, CINDOC (CSIC), CITEFACTOR, COPAC, DIALNET, DICE (CSIC), DOAJ, E-REVISTAS (CSIC), EBSCO PREMIER, ERIH+, GALE CENGAGE LEARNING, IN-RECS, IRESIE, LATINDEX, MIAR, OCLC WORLDCAT, RESH, REDIB, RILM CORE JOURNALS, SUDOC, ULRICHS, ESTA REVISTA
INSTITUCIONAL DE REDIRIS-CONSEJO SUPERIOR DE INVESTIGACIONES CIENTIFICAS Y ES DE ACCESO LIBRE. CREATIVE COMMONS LICENSE 4 O BY 
categoría significativa" (p. 1). Barber y Palacios (2009) reflexionan, también, sobre esta cuestión y opinan que se trata de un "arte en disolución [que] se resiste a ser delimitado por formas y (formalismos) predeterminados, carece de estructuras fijas (...) y asume el conflicto de ser un arte híbrido que se cimienta en la continuidad de su disoluto estar siendo" (p. 9).

Y, en ese entrecruzamiento pluridisciplinar, encontramos la exposición "Paisajes con alma". Esta idea nace y se desarrolla en la Fundación Casa Pintada y Museo Cristóbal Gabarrón con sede en Mula (Murcia, España) y se muestra por primera vez en el Museo del Teatro Romano de Cartagena (Murcia, España) ${ }^{1}$. Dicha muestra plantea la unión de la imagen con la palabra escrita. Partiendo de textos de quince escritores murcianos se pide a otros tantos fotógrafos, también de la Región, que representen visualmente lo escrito: "la literatura es el motor de evocación, donde las artes visuales amplían la descripción haciéndolas visibles" (García, 2017, p. 11). Los textos, dos por autor, evocan paisajes de la Región de Murcia, ya sean naturales, urbanos o espacios íntimos formados por recuerdos o emociones. Se incita entonces a los fotógrafos a mostrar una imagen que se conecte con lo escrito. De este modo, en la muestra se produce una fusión entre ambos lenguajes expresivos lo que invita al espectador a realizar una lectura de la obra expuesta en clave discursiva (el texto) o

\begin{abstract}
"estática (la fotografía). Este hecho deviene en nuevas obras de arte debido a que las imágenes dotan de nuevos significados a los escritos y viceversa. Como se expresa en el prólogo del catálogo de la exposición, "la fotografía se fusiona con la palabra, con los versos o pasajes de la literatura, conformando una nueva obra de arte única y original, que partiendo de la libertad creativa absoluta, cada uno de los autores genera un escenario de reflexión propio" (Gabarrón, 2017, p. 5).
\end{abstract}

Este es el punto de partida de la experiencia educativa que se presenta. Se invitó a un grupo de estudiantes de $4^{\circ}$ de ESO a reinterpretar las lecturas e imágenes de "Paisajes con Alma" desde perspectivas muy variadas y de libre elección por su parte en la que se combinasen expresión corporal, danza, música, arte sonoro, videoarte... Se trataba de trabajos escolares donde, coincidiendo con Berrón (2017) "consideramos que debe primar el proceso de creación y participación de los alumnos y no tanto los resultados" (p. 3), aunque no por ello fuese menos valorable la elaboración final desde el punto de vista estético. Esta experiencia educativa se completaría con una fase de difusión que tiene previsto realizarse en próximas fechas.

\title{
3. Implicaciones educativas de la integración de los lenguajes artísticos
}

La irrupción definitiva de la tecnología en el mundo del arte ayuda sin lugar a dudas a producir sinergias entre los diferentes lenguajes expresivos. Pero, también, contribuye a democratizar los procesos creativos de los artistas permitiendo, cuando se tratan en el aula, abordar los contenidos curriculares relacionados con las artes de una manera diferente. Con el apoyo de la informática podemos sumergir al alumnado en procesos de reflexión-acciónvaloración que desemboquen en la producción de obras artísticas siguiendo técnicas similares a las utilizadas por los creadores contemporáneos.

\footnotetext{
${ }^{1}$ Museo del Teatro Romano de Cartagena. Exposición colectiva. Del 10 de noviembre de 2017 al 15 de enero de 2018. http://Www.teatroromanocartagena.org/

JUAN JESÚS YELO CANO. THE CONTENT OF THIS ARTICLE IS THE SOLE RESPONSIBILITY OF THE AUTHORS. THE REVISTA ELECTRÓNICA DE LEEME AND UNIVERSITAT DE VALĖNCIA ARE NOT LIABLE FOR ANY LEGAL ACTIONS THAT MAY ARISE INVOLVING THE ARTICLE'S CONTENT. REVISTA ELECTRÓNICA DE LEEME -LISTA ELECTRÓNICA EUROPEA DE MÚSICA EN LA SCOPUS, EMERGING CITATION INDEX (CLARIVATE), EBSCO, CINDOC (CSIC), CITEFACTOR, COPAC, DIALNET, DICE (CSIC), DOAU, E-REVISTAS (CSIC), EBSSCO PREMIER, ERIH+, GALE CENGAGE LEARNING, IN-RECS, IRESIE, LATINDEX, MIAR, OCLC WORLDCAT, RESH, REDIB, RILM CORE JOURNALS, SUDOC, ULRICHS, ESTA REVISTA ESTÁ PUBLICADA CON EL APOYO INSTITUCIONAL DE REDIRIS-CONSEJO SUPERIOR DE INVESTIGACIONES CIENTIFICAS Y ES DE ACCESO LIBRE. CREATIVE COMMONS LICENSE 4.O BY
} 
Por una parte, lo que en educación se ha venido llamando centros de interés (Decroly), técnicas Freinet, interdisciplinariedad, etc. junto a los estudios de Vigotsky, Piaget y otros, hoy se reorganiza bajo el concepto "Aprendizaje Basado en Proyectos" (ABP). Este tema excede los límites de este artículo, pero baste decir que los presupuestos que animan esta metodología son los que sustentan la experiencia que se presenta. Muy resumidamente, se trata de que el alumnado vaya construyendo su conocimiento a la vez que desarrolla otras habilidades y competencias que difícilmente encajan en los currículos de las asignaturas oficiales como pueden ser la colaboración, planteamiento y desarrollo de proyectos, y aprendizaje entre iguales entre otros (Galeana, 2006).

Por otra parte, estamos de acuerdo con el Grupo SI(e)TE (2012) a la hora de afirmar que el desarrollo de la creatividad debería ser una de las funciones de la escuela, no sólo en el estudiantado sino también en docentes, equipos directivos y en las propias instituciones educativas. Se comparte con Berrón (2007) el convencimiento de "la escuela del siglo XXI, sin dejar al margen la enseñanza de conceptos y destrezas musicales, debe desarrollar la creatividad de los alumnos si pretende que alcancen una formación integral" (p. 2). Es, por tanto, nuestra intención unir en esta experiencia creatividad, aprendizaje basado en proyectos y arte contemporáneo. El Centro John F. Kennedy para las Artes Escénicas de Washington D.C. define la integración de las artes como "(...) un enfoque de enseñanza en el que los estudiantes construyen y demuestran su conocimiento a través de una forma de arte. Los estudiantes participan en un proceso creativo que conecta una forma de arte con otra asignatura y logran objetivos en ambas" (Silverstein y Layne, 2010, p. 1).

Las experiencias de integración de las artes dentro del mundo educativo son abundantes. Bloomfield (2000) ya afirmaba que "cuando los niños experimentan las artes desde un enfoque integrado su aprendizaje en humanidades, ciencias, tecnología, lectoescritura y aritmética se complementa y enriquece" (p. 1). Un ejemplo de estas iniciativas puede encontrarse en el Whole Schools Project (actualmente, Whole School Initiative) que surgió en Mississipi (Estados Unidos) en 1991. Esta organización nació con la intención de ser un llamamiento a la integración de las artes en el aula y la enseñanza integral en danza, teatro, artes visuales y música. Se trataba de una serie de proyectos de trabajo para el alumnado que integraban las artes visuales y, al menos, otra asignatura entre las de Inglés, Matemáticas y Ciencias Sociales. Algunas de las unidades didácticas pueden consultarse en http://www.mswholeschools.org/. El impacto de esta experiencia fue evaluado por la Mississippi State University y en un informe realizado por Phillpis, Harper, Lee y Boone (s.f.) se publicaron de forma exhaustiva las conclusiones de un estudio realizado entre 2010 y 2011 con 5.500 estudiantes en ocho escuelas públicas y cuatro privadas de Educación Primaria en el estado de Mississippi. Se compararon las calificaciones de los estudiantes en las pruebas estándares estatales de las asignaturas implicadas en el proyecto. Los datos indicaron que las calificaciones del alumnado participante en el modelo de integración de las artes en el currículo fueron significativamente más altas.

Dentro de esa línea, estudios como el de Kindler (2015) defienden la incorporación en el currículo de programas de artes integradas. Organizaciones como ArtStars in Schools en Vancouver (Canadá), el Alabama Institute for Education in the Arts y el ya citado Centro John F. Kennedy para las Artes Escénicas llevan años trabajando en este sentido y colaborando

JUAN JESÚS YELO CANO. THE CONTENT OF THIS ARTICLE IS THE SOLE RESPONSIBILITY OF THE AUTHORS. THE REVISTA ELECTRÓNICA DE LEEME AND UNIVERSITAT DE VALĖNCIA ARE NOT LIABLE FOR ANY LEGAL ACTIONS THAT MAY ARISE INVOLVING THE ARTICLE'S CONTENT. REVISTA ELECTRÓNICA DE LEEME - LSTA ELECTRÓNICA EUROPEA DE MÚSICA EN LA EDUCACIÓN-. HTTP://OJS.UV.ES/INDEX.PHP/LEEME/INDEX ISSN: 1575-9563 EDITORES: UNIVERSIDAD DE VALENCIA Y JESÚS TEJADA GIMÉNEZ. VISIBILIDAD DE ESTA REVISTA: SCOPUS, EMERGING CITATION INDEX (CLARIVATE), EBSCO, CINDOC (CSIC), CITEFACTOR, COPAC, DIALNET, DICE (CSIC), DOAJ, E-REVISTAS (CSIC), EBSCO PREMIER, ERIH+, GALE CENGAGE LEARNING, IN-RECS, IRESIE, LATINDEX, MIAR, OCLC WORLDCAT, RESH, REDIB, RILM CORE JOURNALS, SUDOC, ULRICHS, ESTA REVISTA ESTÁ PUBLICADA CON EL APOYO INSTITUCIONAL DE REDIRIS-CONSEJO SUPERIOR DE INVESTIGACIONES CIENTIFICAS Y ES DE ACCESO LIBRE. CREATIVE COMMONS LICENSE 4.O BY 
activamente con centros educativos en proyectos que integran las artes. Estas instituciones, al igual que la Whole School Initiative ya mencionada, contemplan el desarrollo de unidades didácticas que implican secuencias de enseñanza que incluyen actividades relacionadas con las artes y otras materias con un tratamiento interdisciplinar. Estas organizaciones buscan la implicación de profesorado, artistas, familias y comunidad, a través de actuaciones en centros escolares, residencias de artistas, ayudas a escuelas, cursos de formación para profesorado, galerías de exposiciones y programas para arte en familia. En un reciente informe de la ArtStars (2017) se destacó el gran alcance e impacto en las escuelas de la zona: 1.997 espectáculos en más de 200 localidades de la región, 2.822 alumnos expusieron en galerías de arte, 61 distritos escolares (de 62) recibieron ayudas para desarrollar actividades relacionadas con el mundo del arte.

Otro programa interesante es el Project Zero (http://www.pz.harvard.edu/) desarrollado en la Universidad de Harvard. Este programa abarca numerosos planes que investigan y analizan diversas propuestas de interdisciplinariedad llevadas a cabo por profesorado de secundaria en distintos contextos educativos, produciendo materiales de gran ayuda para docentes. Uno de estos proyectos es el Artful Thinking que se centra en mirar e interpretar el arte a través de una serie de rutinas básicas integradas en la vida cotidiana del aula. Estas estrategias "pueden ser estructuras simples, como leer un texto y responder las preguntas al final del capítulo, o pueden estar diseñadas para promover el pensamiento de los alumnos, como preguntarle a los alumnos qué saben, qué quieren saber y lo que han aprendido en una unidad didáctica” (Thisman y Palmer, 2007, p. 14). Estos investigadores demuestran en su detallado estudio estadístico cómo esta experiencia "causa una temprana e intensa aceleración en el índice de conceptualización del alumnado".

Relacionado con los trabajos anteriores y bajo la supervisión de Rojas (2016), el Consejo Nacional de la Cultura y las Artes de Chile edita una serie de cuadernos dentro de la colección "Caja de herramientas para la educación artística" invitando a docentes, artistas, gestores culturales y estudiantes a la "reflexión colectiva sobre el valor y los aportes de la educación artística y cultural en la construcción de una educación de calidad más integral" (p. 8). Esa misma publicación señala como beneficio de la interdisciplinariedad en las artes el de "construir un conocimiento complejo, dinámico y localizado" (p. 32). Uno de los proyectos presentados como buena práctica fue el desarrollado en un colegio de Santiago de Chile desde el año 2011. La participación en este plan era voluntaria para el alumnado y en él "pueden elegir entre diferentes 'menciones' [Historia, Lenguaje, Ciencias y Artes] desde las cuales plantear sus proyectos de investigación. Si bien cada mención se refiere a un área específica, existe la posibilidad de resolver inquietudes a partir del cruce entre disciplinas" (p. 36). El proyecto constaba de tres fases: configuración de los grupos de trabajo, proceso de investigación y difusión de los resultados. Era en esta última etapa en la que las artes jugaban su papel, ya que para la "transferencia del conocimiento generado [se elaboran] ensayos, cortometrajes, obras de arte, entre otros" (p. 37). El profesorado responsable del proyecto destacaba como logro "la calidad del nuevo conocimiento generado por los estudiantes participantes" (Mayorga, 2014, p.18). 
Otro proyecto interesante ha sido el desarrollado durante 2017 en dos colegios públicos y una casa-hogar en Uruguay. Gadea (2018), profesor de danza en Primaria en esos centros, se ha dado cuenta de que "los chicos tenían pocas opciones para expresase en varios formatos". A partir de esta idea, ha construido una propuesta que integra en un solo espectáculo danza, teatro y artes plásticas. Posteriormente, se incorpora la idea de utilizar materiales de reciclaje para la confección de decorados y vestuario con lo que se pretende "buscar un cruce entre las artes y la educación y [el reciclaje] resultó un buen hilo conductor entre las distintas artes. Además, trae algo de su cotidianidad, porque los niños se dan cuenta de que el arte puede estar en eso que es parte de su día a día". La propuesta, premiada y subvencionada por el Ministerio de Educación y Cultura de Uruguay, fue todo un éxito entre los participantes, ya que como dice Gadea,

\begin{abstract}
"funcionaba con todas las edades: tanto al niño de siete como al de 12 años les gustaba moverse y saltar a la cuerda. Pensamos que capaz que la propuesta debería ser diferente para las distintas edades, pero nos sorprendió cómo todos tienen esa necesidad de expresarse físicamente; no hubo ni un lugar donde no quisieran participar, incluso, grupos que no habían logrado la conexión entre ellos lo hicieron por medio del baile" (p. 1).
\end{abstract}

Sterman (2018), vicedirectora de educación y vicedirectora del Partnership for 21st Century Learning en Estados Unidos, indica cómo "la incorporación de las artes en el currículo puede mejorar el éxito de los estudiantes" (p. 31). Para realizar esta afirmación se apoya en estudios estadísticos sobre resultados académicos, como el realizado por Stoelinga, Silk, Reddy y Rhaman (2015).

En España, se realizan experiencias educativas que promueven en el alumnado la creación de obras de música experimental partiendo de las artes plásticas. Un proyecto de estas características es el desarrollado por Yelo (2012) en el que diseña un modelo didáctico basado en procesos de escucha-reflexión-acción-valoración similares a los que realizan algunos compositores contemporáneos. Partiendo de la escucha atenta de los sonidos del entorno se invitó al alumnado a reflexionar sobre sus significados para el individuo y para la comunidad para a continuación utilizarlos en una primera propuesta sonora tomando como pretexto una fotografía. La premisa para el alumnado consistió en la creación de un paisaje sonoro acorde a la imagen seleccionada. Tras una valoración del resultado obtenido, se repitió el proceso teniendo ahora como elemento generador de la composición una pintura del Museo de Bellas Artes de Murcia (España). Por último, manipulando los sonidos en un editor de audio se realizó nuevamente el procedimiento partiendo ahora de una pintura abstracta. La exposición simultánea de las creaciones en el propio museo y en el Laboratorio de Arte Joven proporcionó una dimensión social al proyecto.

Utilizando el mismo diseño metodológico, Yelo (2017) desarrolló otra experiencia, pero partiendo ahora de textos. Las "Ciudades Invisibles" de Italo Calvino se convirtieron en el motor generador de la creación que el alumnado realizaba de forma individual. En un primer momento, se crearon obras plásticas basadas en la descripción que el escritor italiano hacía de las ciudades fantásticas para concluir con una composición de música experimental que fuese lo más fiel posible al espíritu del relato. 


\section{La experiencia educativa "Paisajes con alma"}

Silverstein y Layne (2010, p.9) proporcionan una checklist para verificar si simplemente se estaba incluyendo el arte en nuestras clases o, realmente, lo estábamos integrando en la práctica diaria. El proyecto "Paisajes con alma" respondía a sus preguntas afirmativamente, de manera que cumplía con los siguientes requisitos:

1.- Eran evidentes los principios del aprendizaje constructivista (colaborativo, experiencial, resolución de problemas...).

2.- El alumnado:

- Estaba comprometido a construir y comprender, no sólo a memorizar.

- Construía y demostraba su comprensión a través de una forma de arte.

- Estaba involucrado en un proceso de creación de algo original en lugar de copiar.

- Revisaba sus producciones.

3.- La forma de arte escogida para el trabajo estaba conectada con contenidos de otras asignaturas (Tecnología, Comunicación Audiovisual, Lengua) y con otras necesidades no presentes explícitamente en el currículo como era la educación emocional. Ambas conexiones salían reforzadas en el proyecto.

4.- Los objetivos específicos de la propuesta concernían tanto a la forma de arte utilizada por los estudiantes como a las otras asignaturas curriculares. El desafío para el alumnado era avanzar en el modo de expresión artística escogido y en los medios materiales necesarios para llevarla a cabo.

Esta experiencia tenía como uno de sus pilares fundamentales el desarrollo de la creatividad del alumnado. Si nos basamos en las indicaciones del Grupo SI(e)TE (2012, pp. 9 y ss.) puede comprobarse que este proyecto cumple con las cuatro condiciones necesarias para ser creativo. En primer lugar, el resultado era un producto nuevo que respondía a la utilización del pensamiento creativo (era original) en connivencia con el pensamiento crítico (se actuaba de acuerdo a unos criterios). En segundo lugar, el proceso era la parte verdaderamente importante de la experiencia. En tercer lugar, la personalidad del alumno debía reflejarse en su trabajo; se trataba de un compromiso personal que ponía en juego las emociones y la inteligencia personal e interpersonal. Por último, se ponían en marcha contextos creativos donde se favorecía la participación y la libre expresión de ideas, expuestos a la crítica, pero sin miedo al rechazo, donde el profesorado se convertía en catalizador, mediador, potenciador, y no en la figura autoritaria poseedora de la verdad absoluta.

\subsection{Objetivos}

Los objetivos de este proyecto se concretaron en:

a) Introducir al alumnado en los procesos de composición de la obra artística para que experimentasen los momentos de reflexión-acción-valoración en la realización de la tarea creativa propuesta. 
b) Proporcionar al estudiantado herramientas que le ayudasen a entender el complejo mundo del arte contemporáneo en varias de sus facetas.

c) Fomentar en el alumnado la conciencia de pertenencia a una sociedad a la que debían contribuir en un futuro no tan lejano, bien como creadores, bien como espectadores conscientes del valor del arte contemporáneo.

\subsection{Participantes}

La experiencia presentada se desarrolló durante el segundo trimestre del curso 2017/18 en un instituto de Educación Secundaria de la ciudad de Murcia. El alumnado pertenecía a la asignatura de "Artes Escénicas y Danza", una de la optativas de $4^{\circ}$ de la ESO ofertadas en dicho centro. A ella asistían adolescentes de 15-16 años provenientes de cuatro grupos diferentes y dentro de ellos, podían pertenecer al subgrupo bilingüe o al ordinario. En total, 12 alumnas (todas chicas) realizaron el trabajo propuesto por el docente.

\subsection{Desarrollo de la experiencia}

En una fase previa al trabajo con el alumnado, se contactó con uno de los comisarios de la exposición para comunicarle el proyecto de utilizar textos y fotografías de la muestra como material para trabajar en el aula de música. Entusiasmado con la propuesta "Paisajes con alma en el centro escolar", el comisario facilitó varios catálogos de la exposición, así como la versión en pdf para poder imprimir los textos con facilidad y distribuirlos al alumnado.

\section{Fase 1. Reflexión. Acción}

La propuesta de trabajo fue explicada al alumnado. Para introducir la cuestión, el docente comentó cómo en la exposición "Paisajes con alma" se presentaban textos de escritores murcianos y, a su lado, se colocaron las imágenes con las que fotógrafos, también de la Región, habían interpretado los escritos. A continuación, se propuso al estudiantado la realización de un trabajo en el que, partiendo de los pasajes y la imagen asociada, realizasen una nueva interpretación en un lenguaje artístico diferente y lo registrasen en un soporte, preferentemente audio o vídeo. Se distribuyeron copias de todos los escritos que formaban parte de la exposición: eran 30 textos (dos por autor) entre los que se encontraban 14 poemas (algunos inéditos) y 16 fragmentos en prosa de libros o diarios personales. Tras una detenida lectura, individualmente o por parejas, el alumnado eligió un documento con el que trabajar. La única premisa era que debían escoger uno que de alguna manera les implicase emocionalmente.

Después se le proporcionaba la imagen con la que el fotógrafo había interpretado ese escrito para mostrar al alumnado cómo "distintas formas de trabajar y de mirar, constituyen un conjunto, una experiencia total y de creación" (García, 2017, p. 11). A continuación, se invitaba al alumnado a que comenzase a trabajar con el texto y la fotografía y buscase un significado a ambos que pudieran reducir a una o dos palabras, como por ejemplo soledad, orgullo, infancia o paseo. Es en este momento cuando se les pidió que encontrasen un nexo entre sus emociones y la obra escogida y que relacionasen esa interpretación con algo cercano a su experiencia de vida.

JUAN JESÚS YELO CANO. THE CONTENT OF THIS ARTICLE IS THE SOLE RESPONSIBILITY OF THE AUTHORS. THE REVISTA ELECTRÓNICA DE LEEME AND UNIVERSITAT DE VALĖNCIA ARE NOT LIABLE FOR ANY LEGAL ACTIONS THAT MAY ARISE INVOLVING THE ARTICLE'S CONTENT. REVISTA ELECTRÓNICA DE LEEME -LISTA ELECTRÓNICA EUROPEA DE MÚSICA EN LA EDUCACIÓN-. HTTP://OJS.UV.ES/INDEX.PHP/LEEME/INDEX ISSN: 1575-9563 EDITORES: UNIVERSIDAD DE VALENCIA Y JESÚS TEJADA GIMENEZ. VISIBILIDAD DE ESTA REVISTA. CENGAGE LEARNING, IN-RECS, IRESIE, LATINDEX, MIAR, OCLC WORLDCAT, RESH, REDIB, RILM CORE JOURNALS, SUDOC, ULRICHS, ESTA REVISTA ESTÁ PUBLICADA CON EL APOYO INSTITUCIONAL DE REDIRIS-CONSEJO SUPERIOR DE INVESTIGACIONES CIENTIFICAS Y ES DE ACCESO LIBRE. CREATIVE COMMONS LICENSE 4.O BY 
Debían decidir, también, el modo en el que iban a reinterpretar las palabras del escritor y determinar de qué manera registrar su proposición (vídeo, audio, etc.), para pasar a elaborar un plan de trabajo que incluyese una temporalización y los recursos materiales a utilizar. Sus propuestas debían demostrar que habían llevado a cabo un trabajo de análisis y apropiación de los significados de las obras artísticas ya creadas y establecer con ellas un vínculo que sumase una nueva dimensión a las mismas.

\section{Fase 2. Valoración}

Finalmente, se formaron cuatro parejas de chicas y otras cuatro alumnas trabajaron en solitario. Las participantes presentaron al resto de la clase los textos escogidos, se mostraron las imágenes y comentaron su proyecto. El resto de la clase, estuviesen realizando o no la propuesta, hacía aportaciones y sugerencias que permitiesen enriquecer algunas de ellas. Estas aportaciones de los compañeros eran de tipo conceptual, pero también las había de tipo técnico: cómo utilizar la cámara de vídeo, hacer el montaje, utilizar el editor de audio, alguna aplicación para móvil, etc.

En esta fase, el papel del docente consistía en ser el facilitador de los procesos, coordinando los turnos de palabra e interviniendo sólo para ayudar a que el contexto fuese lo más distendido posible y el alumnado pudiese expresarse libremente. Además, vigilaba que las críticas se hiciesen desde un punto de vista constructivo y evitaba en todo momento que las alumnas que exponían sus propuestas se sintieran juzgadas por sus compañeras.

\section{Fase 3. Reflexión. Acción}

Con las aportaciones de los compañeros y una vez redefinido el trabajo a producir, las alumnas proceden a realizarlo. Para la elaboración del proyecto escogido, deben acudir a competencias desarrolladas en otras disciplinas. Por ejemplo, si construyen un paisaje sonoro a partir de grabaciones de campo deben utilizar un editor de audio cuyo manejo han aprendido en la asignatura de Tecnología. Si el formato escogido es video deben poner en práctica las nociones sobre imagen fija y en movimiento trabajadas en las asignaturas de Educación Plástica y Visual, así como la edición de video o la técnica del stop motion. La mayoría han de hacerlo en horario extraescolar y fuera del centro. Algunos de ellos incluso implican a familiares y amigos en la confección, aumentando si cabe la implicación personal en el proyecto.

\section{Fase 4. Reflexión. Valoración.}

Por último, una vez finalizado el trabajo se pide al alumnado que detalle qué pasos ha seguido para realizarlo y cuál es el significado de su creación que, obviamente, ha de estar en consonancia con el texto y/o la imagen generadora. Esta justificación y la exposición de la obra a los compañeros dio paso a una nueva ronda de valoraciones con la finalidad de que el propio grupo de clase evaluase las elaboraciones artísticas resultantes. 


\section{Fase 5. Difusión de las creaciones}

Una vez terminado el trabajo, se comentó con el comisario de fotografía la posibilidad de realizar una difusión de las creaciones de las alumnas. Después de ver las producciones realizadas, se llegó al acuerdo de incorporarlas al proyecto de la Fundación Casa Pintada cuando se exhibiese en otro lugar, ya que, como indica Gabarrón (2017), desde el principio estaba previsto que el proyecto "Paisajes con alma" inicie "una gira, que viajará más allá de sus fronteras [de la Región de Murcia]” (p. 5).

Esta fase está aún pendiente de realizar y el formato escogido consiste en incorporar unos códigos QR a la exposición que remitan directamente a las obras de las alumnas alojadas en la red. Otra opción que se baraja, siempre que las condiciones lo permitan, es reinterpretar algunas de las obras en directo, bien los días de inauguración, bien en sesiones específicas con asistencia de público.

\section{Resultados}

En total, se presentan ocho creaciones. En la Tabla 1, pueden apreciarse las diferentes características y formatos de las obras realizadas por las alumnas. Dichas creaciones pueden contemplarse en https://goo.gl/m5Fe7h

Tabla 1. Análisis de las creaciones realizadas por las alumnas

\begin{tabular}{|c|c|c|c|c|c|}
\hline ESTUDIANTES & OBRA & FORMATO & $\begin{array}{l}\text { LENGUAJE } \\
\text { ARTÍSTICO }\end{array}$ & IMAGEN & AUDIO \\
\hline E1 y E2 & V1 & Vídeo & $\begin{array}{l}\text { Cine mudo en } \\
\text { color }\end{array}$ & $\begin{array}{l}\text { Imagen en } \\
\text { movimiento }\end{array}$ & Música de fondo \\
\hline E3 & $\mathrm{V} 2$ & Vídeo & $\begin{array}{l}\text { Gimnasia } \\
\text { rítmica }\end{array}$ & $\begin{array}{l}\text { Imagen en } \\
\text { movimiento }\end{array}$ & $\begin{array}{l}\text { Estudio en } D \text { menor de } O p \text {. } \\
46 n^{\circ} 14 \text { de Stephen Heller } \\
\text { interpretado por una amiga de } \\
\text { la alumna y grabada ex } \\
\text { profeso para el vídeo }\end{array}$ \\
\hline E4 y E5 & V3 & Vídeo & $\begin{array}{l}\text { Cine mudo en } \\
\text { color }\end{array}$ & $\begin{array}{l}\text { Imagen en } \\
\text { movimiento }\end{array}$ & $\begin{array}{l}\text { Preludio de la suite } n^{o} 1 \text { de } \mathrm{J} \text {. } \\
\text { S. Bach interpretado por la } \\
\text { alumna y grabada ex profeso } \\
\text { para el vídeo }\end{array}$ \\
\hline E6 & V4 & Vídeo & Videoarte & $\begin{array}{l}\text { Imagen en } \\
\text { movimiento }\end{array}$ & Lluvia \\
\hline E7 & V5 & Vídeo & Arte sonoro & $\begin{array}{l}\text { Imágenes fijas } \\
\text { (sonogramas) }\end{array}$ & $\begin{array}{l}\text { Audio grabado ex profeso } \\
\text { para el vídeo }\end{array}$ \\
\hline E8 y E9 & V6 & Vídeo & Stop motion & $\begin{array}{l}\text { Imágenes fijas } \\
\text { (fotografías) }\end{array}$ & ---- \\
\hline E10 y E11 & V7 & Vídeo & $\begin{array}{l}\text { Cine en } \\
\text { blanco y negro }\end{array}$ & $\begin{array}{l}\text { Imágenes fijas } \\
\text { (fotografías) + } \\
\text { imagen en } \\
\text { movimiento }\end{array}$ & $\begin{array}{l}\text { Música de fondo }+ \text { voz en off } \\
\text { con el texto original }\end{array}$ \\
\hline E12 & A1 & Audio & $\begin{array}{l}\text { Música } \\
\text { concreta }\end{array}$ & ---- & $\begin{array}{l}\text { Música concreta: paisaje } \\
\text { sonoro narrado en el texto }\end{array}$ \\
\hline
\end{tabular}

Fuente: Elaboración propia

Si atendemos a la utilización del audio, la música de fondo de las obras V1 y V7 se obtiene en Internet de páginas libres de derechos de autor. Las composiciones V2 y V3 incluyen

JUAN JESÚS YELO CANO. THE CONTENT OF THIS ARTICLE IS THE SOLE RESPONSIBILITY OF THE AUTHORS. THE REVISTA ELECTRÓNICA DE LEEME AND UNIVERSITAT DE VALĖNCIA ARE NOT LIABLE FOR ANY LEGAL ACTIONS THAT MAY ARISE INVOLVING THE ARTICLE'S CONTENT. REVISTA ELECTRÓNICA DE LEEME - LISTA ELECTRÓNICA EUROPEA DE MÚSICA EN LA EDUCACIÓN-. HTTP://OJS.UV.ES/INDEX.PHP/LEEME/INDEX ISSN: 1575-9563 EDITORES: UNIVERSIDAD DE VALENCIA Y JESÚS TEJADA GIMÉNEZ. VISIBILIDAD DE ESTA REVISTA: SCOPUS, EMERGING CITATION INDEX (CLARIVATE), EBSCO, CINDOC (CSIC), CITEFACTOR, COPAC, DIALNET, DICE (CSIC), DOAJ, E-REVISTAS (CSIC), EBSCO PREMIER, ERIH+, GALE CENGAGE LEARNING, IN-RECS, IRESIE, LATINDEX, MIAR, OCLC WORLDCAT, RESH, REDIB, RILM CORE JOURNALS, SUDOC, ULRICHS, ESTA REVISTA ESTÁ PUBLICADA CON EL APOYO INSTITUCIONAL DE REDIRIS-CONSEJO SUPERIOR DE INVESTIGACIONES CIENTIFICAS Y ES DE ACCESO LIBRE. CREATIVE COMMONS LICENSE 4.O BY 
grabaciones de música clásica interpretadas ex profeso para el vídeo. El audio de A1 es una obra de música concreta que describe el paisaje sonoro recogido en el escrito de partida mientras que en V4 se trata del sonido de la lluvia (nombre del poema recreado). El empleo de la voz humana lo encontramos en V7 y en V5, si bien esta última obra se adentra en los terrenos del arte sonoro: se distorsiona tanto el audio que resulta imposible reconocer que suena una voz humana.

Si se presta atención al uso del vídeo, de los siete trabajos en este formato, la mayoría confían gran parte del mensaje a la expresividad de la imagen en movimiento (V1, V2, V3 y V4), mientras que los que utilizan la imagen fija son V5 y V6 y sólo V7 utiliza una técnica mixta, reforzada por el uso del blanco y negro. La combinación del uso del audio y de la imagen en los siete vídeos da resultados muy variables, desde producciones más o menos convencionales (pero no por ello menos expresivas) hasta creaciones muy cercanas al videoarte (V4 y V5). La obra que utiliza solamente audio (A1) es un ejemplo paradigmático de música concreta, narrando sólo con sonidos el paisaje sonoro que se intuye en las líneas del texto escogido.

Si se analiza el contenido de las obras, puede observarse cómo ha quedado plasmada la conexión del trabajo con las emociones y la implicación personal de las alumnas. En la fase 4 (reflexión, valoración), se pudo escuchar sus explicaciones y comprobar cómo en sus producciones aparecieron estos elementos en mayor o menor medida. En V7, acudieron a recuerdos de la infancia, utilizando fotografías de su propia niñez. En otros casos, se escogió una actividad extraescolar realizada durante años como hilo conductor de la narración: equitación y violonchelo (V3) o gimnasia rítmica (V2). Además, la autora de V2 escogió ese texto porque conocía personalmente a la fotógrafa que lo había interpretado visualmente. En V6, se acudió a imágenes realizadas desde la azotea de su vivienda para mostrarnos paisajes muy significativos para una de las creadoras. En V4, la conexión (según explicó la alumna) se encuentra directamente relacionada con momentos o situaciones de desánimo vividas que le acudieron a la memoria cuando leyó el texto. El arte sonoro reflejado en V5 sea, tal vez, el que estaba menos vinculado con las emociones, pero sí expresaba el deseo de su autora de experimentar con nuevas herramientas, ya que había descubierto hacía poco la aplicación de móvil que usó para la creación y posterior lectura de los sonogramas que componen la obra. Por último, en otras creaciones se implicó directamente a la familia en la realización, apareciendo como actor un hermano pequeño (V1) o pidiendo que produjeran los sonidos que necesitaba para componer el paisaje sonoro deseado (A1).

Los resultados de este trabajo han superado las expectativas iniciales. En cuanto al primer objetivo de introducir al alumnado en los procesos de composición de la obra artística, el proceso de trabajo de reflexión-acción-valoración se ha mostrado muy eficiente en vista de las creaciones realizadas por las alumnas. Además, en una encuesta (ver Anexo I) contestada al finalizar la experiencia $(n=11)$, todas se muestran conscientes de que han seguido un proceso para realizar el trabajo: 8 manifiestan haber realizado una planificación previa a la grabación de la obra, 2 reconocen que su método ha sido la improvisación y 1 ha utilizado el ensayo-error. 10 de ellas creen entender mejor los procesos creativos de los artistas después de haber realizado la experiencia.

JUAN JESÚS YELO CANO. THE CONTENT OF THIS ARTICLE IS THE SOLE RESPONSIBILITY OF THE AUTHORS. THE REVISTA ELECTRÓNICA DE LEEME AND UNIVERSITAT DE VALĖNCIA ARE NOT LIABLE FOR ANY LEGAL ACTIONS THAT MAY ARISE INVOLVING THE ARTICLE'S CONTENT. REVISTA ELECTRÓNICA DE LEEME -LISTA ELECTRÓNICA EUROPEA DE MÚSICA EN LA EDUCACIÓN-. HTTP://OJS.UV.ES/INDEX.PHP/LEEME/INDEX ISSN: 1575-9563 EDITORES: UNIVERSIDAD DE VALENCIA Y JESÚS TEJADA GIMÉNEZ. VISIBILIDAD DE ESTA REVISTA: CENGAGE LFARNING, IN-RECS, IRESIE, (CLARIVATE), EBSCO, CINDOC (CSIC), CITEFACTOR, COPAC, DIALNET, DICE (CSIC), DOAJ, E-REVISTAS (CSIC), EBSCO PREMIER, ERIH , GALE INSTITUCIONAL DE REDIRIS-CONSEJO SUPERIOR DE INVESTIGACIONES CIENTIFICAS Y ES DE ACCESO LIBRE. CREATIVE COMMONS LICENSE 4.0 BY 
En las respuestas a la pregunta "¿qué dirías que ha aportado este trabajo a tu formación como persona?" todas las alumnas, excepto una que responde nada, hacen alusión de alguna u otra manera a lo que han aprendido sobre creatividad y a cómo ha mejorado su comprensión sobre el trabajo de los artistas.

En relación con el segundo objetivo de proporcionar al alumnado el uso de herramientas para comprender mejor el arte contemporáneo, 10 de las alumnas han considerado interesante y/o agradable producir una obra artística basada en otras previas mientras que a una le ha resultado una tarea indiferente. Por otra parte, 10 han terminado contentas con su elaboración artística y son conscientes de que han creado arte; una de ellas está incluso algo triste "al descubrir que lo que [yo] había imaginado no era tan sencillo de plasmar como creía". La última alumna reconoce que ha acabado cansada, ya que le "ha costado mucho trabajo". En otro momento de la encuesta, varias alumnas reconocen haber aprendido sobre el uso de herramientas que les permitirán poder expresar sus ideas de una manera artística.

Con respecto al tercer objetivo, las estudiantes son muy conscientes de que han creado una obra artística y que el esfuerzo invertido ha sido grande. De alguna manera quieren ver reconocido su labor y, por ello, ninguna duda en responder afirmativamente cuando se les propone que su obra sea expuesta al público como parte de la exposición "Paisajes con alma". Opinan que han hecho algo "que puede aportar ideas y cultura" y se sienten orgullosas e ilusionadas de dar visibilidad a su trabajo. Incluso a una de ellas le parece "interesante que (...) otras personas puedan compartir su opinión sobre lo que hemos hecho", de acuerdo con la información recopilada.

\section{Conclusiones}

El trabajo de las disciplinas artísticas en la educación escolar, ya sea en el ámbito plástico, musical, dramático, dinámico, tecnológico, etc., tiene un gran valor para el desarrollo del individuo. La integración de las artes como uno de los pilares básicos de la escuela representa una de las soluciones a los retos a los que se enfrenta la educación del siglo XXI.

La experiencia "Paisajes con alma" entiende que la puesta en práctica de una educación que contemple una visión holística de las artes debe sentar sus bases en tres premisas. En primer lugar y en concordancia con Berrón (2017), es importante valorar el proceso más que el resultado, pues es la experiencia generada por el acto de la creación artística la que produce el aprendizaje, más allá de la calidad artística de las obras producidas. Por otra parte, es necesario el uso de estrategias metodológicas basadas en la interdisciplinariedad, como se aprecia también en las unidades didácticas desarrolladas en los centros de la Whole School Initiative, ArtStars in Schools y Project Zero. Por último, experiencias como la de Mayorga (2014) y los centros que practican el Artful Thinking, coinciden con "Paisajes con alma" en la utilización del aprendizaje basado en proyectos como herramienta para que el alumnado desarrolle habilidades que le permitan buscar soluciones creativas a la cuestión planteada.

A pesar de priorizar el proceso frente al resultado, en experiencias como la presentada, las obras producidas por las alumnas, los procedimientos seguidos para su realización y los JUAN JESÚS YELO CANO. THE CONTENT OF THIS ARTICLE IS THE SOLE RESPONSIBILITY OF THE AUTHORS. THE REVISTA ELECTRÓNICA DE LEEME AND UNIVERSITAT DE VALËNCIA ARE NOT LIABLE FOR ANY LEGAL ACTIONS THAT MAY ARISE INVOLVING THE ARTICLE'S CONTENT. REVISTA ELECTRÓNICA DE LEEME -LISTA ELECTRÓNICA EUROPEA DE MÚSICA EN LA SCOPUS, EMERGING CITATION INDEX (CLARIVATE), EBSCO, CINDOC (CSIC), CITEFACTOR, COPAC, DIALNET, DICE (CSIC), DOAJ, E-REVISTAS (CSIC), EBSCO PREMIER, ERIH+, GALE CENGAGE LEARNING, IN-RECS, IRESIE, LATINDEX, MIAR, OCLC WORLDCAT, RESH, REDIB, RILM CORE JOURNALS, SUDOC, ULRICHS, ESTA REVISTA ESTÁ PUBLICADA CON EL APOYO INSTITUCIONAL DE REDIRIS-CONSEJO SUPERIOR DE INVESTIGACIONES CIENTIFICAS Y ES DE ACCESO LIBRE. CREATIVE COMMONS LICENSE 4.0 BY 
comentarios finales de las participantes permiten hacer dos afirmaciones. Por un lado, han implicado en su trabajo sus conocimientos sobre el uso de las tecnologías de audio y vídeo, desarrollando lo que ya sabían, pero también aprendiendo nuevas utilidades. Por otra parte, ha existido una implicación de tipo emocional que ha posibilitado verter sus sentimientos en las elaboraciones artísticas realizadas. Se han producido por tanto beneficios de tipo personal para las estudiantes, ya que han desarrollado muy adecuadamente varias de sus competencias, en especial la relacionada con la conciencia y expresión culturales, aunque también las que tienen que ver con la inteligencia emocional.

Gozar de la experiencia estética de entrar en contacto con lo bello a través del desarrollo de proyectos de creación artística en nuestras aulas debe permitir al alumnado desarrollar su creatividad a través de dinámicas de trabajo que incluyan el uso de las herramientas tecnológicas. Esto le posibilitará conocer los procedimientos que utilizan los artistas para sus creaciones y, sin lugar a dudas, los convertirá en personas más capaces de comprender el lenguaje contemporáneo del arte.

\section{Referencias}

Barber, Ll. y Palacios, M. (2009). La mosca tras la oreja. De la música experimental al arte sonoro en España. Madrid: Fundación Autor.

Berrón, E. (2017). Integración creativa de la música Pop-Rock en el aula. Revista Electrónica de LEEME, 39, 1-20. doi: 10.7203/LEEME.39.10070

Bloomfield, A. y Childs, J. (2000). Teaching Integrated Arts in the Primary School. Nueva York: Routledge.

Gabarrón-Cabero, C. (2017). Textos introductorios al catálogo. En VV.AA. Paisajes con alma (p. 5). Murcia: Fundación Cristóbal Gabarrón.

Gadea, G. (2018). De acá para allá. La diaria (6-I-18). Recuperado de https://findesemana.ladiaria.com.uy/

Galeana, L. (2006). Aprendizaje basado en proyectos. Investigación en Educación a Distancia. Revista Digital. Recuperado de http://ceupromed.ucol.mx/

García-Sandoval, J. (2017). Alma de lo interno y externo. En VV.AA., Paisajes con alma (p. 11). Murcia: Fundación Cristóbal Gabarrón.

Grupo SI(e)TE (2012). Creatividad, educación e innovación: emprender la tarea de ser autor y no sólo actor de sus propios proyectos. Revista de Investigación en Educación, 10 (1), 7-29. Recuperado de http://reined.webs.uvigo.es/

Kindler, A. M. (2015). A Review of Rationales for Integrated Arts Programs. Studies in Art Education, 29, 52-60. Doi: 10.1080/00393541.1987.11650668

JUAN JESÚS YELO CANO. THE CONTENT OF THIS ARTICLE IS THE SOLE RESPONSIBILITY OF THE AUTHORS. THE REVISTA ELECTRÓNICA DE LEEME AND UNIVERSITAT DE VALĖNCIA ARE NOT LIABLE FOR ANY LEGAL ACTIONS THAT MAY ARISE INVOLVING THE ARTICLE'S CONTENT. REVISTA ELECTRÓNICA DE LEEME - LISTA ELECTRÓNICA EUROPEA DE MÚSICA EN LA EDUCACIÓN-. HTTP://OJS.UV.ES/INDEX.PHP/LEEME/INDEX ISSN: 1575-9563 EDITORES: UNIVERSIDAD DE VALENCIA Y JESÚS TEJADA GIMÉNEZ. VISIBILIDAD DE ESTA REVISTA: SCOPUS, EMERGING CITATION INDEX (CLARIVATE), EBSCO, CINDOC (CSIC), CITEFACTOR, COPAC, DIALNET, DICE (CSIC), DOAJ, E-REVISTAS (CSIC), EBSCO PREMIER, ERIH+, GALE INSTITUCIONAL DE REDIRIS-CONSEJO SUPERIOR DE INVESTIGACIONES CIENTIFICAS Y ES DE ACCESO LIBRE. CREATIVE COMMONS LICENSE 4.0 BY 
Mayorga, R. (2012). Proyectos ganadores de profesores de instituciones pertenecientes a la red de experiencias laborales de la Universidad Alberto Hurtado. Cuadernos de Educación, 60, 1620. Recuperado de http://mailing.uahurtado.cl/

Phillpis, J., Harper, J., Lee, K. y Boone, E. (s.f.). Arts Integration \& the Mississippi Art's Commission's Whole Schools Initiative. Mississippi: Mississippi State University. Recuperado de http://www.mswholeschools.org/

Molina, M. (Ed.) (2017). ¡Chum, Chum, Pim, Pam, Pum, Olé!. Valencia: Laboratorio de Creaciones Intermedia.

Rojas, P. (Ed.) (2016). Caja de herramientas para la educación artística. Cuaderno 3. Santiago de Chile: Consejo Nacional de la Cultura y las Artes. Recuperado de https://www.cultura.gob.cl/

Silverstein, L. y Layne, S. (2010). Defining Arts Integration. Washington: The John F. Kennedy of the Performing Arts. Recuperado de http://www.kennedy-center.org/education/

Rudi, J. (2009). Editorial. Organised sound, 14 (I), 1-2. doi: 10.1017/S1355771809000016

Sterman, C. (2018). Arts integration improve school culture and student success. Principal. Comprehensive Learning, vol. 97 (3), 30-31. Recuperado de https://www.naesp.org/principal

Stoelinga, S., Silk, Y., Reddy, P. y Rhaman, N. (2015). Final Evaluation Report. Turnarpound Arts Initiative. Washington: Committee on the Arts and the Humanities. Recuperado de http://turnaroundarts.kennedy-center.org/

Takkal, A. y Rubio, A. (2017). Arte sonoro y la dialéctica entre las artes y el arte. En M. Molina (ed.), ¡Chum, Chum, Pim, Pam, Pum, Olé! (pp. 24-32). Valencia: Laboratorio de Creaciones Intermedia.

Tishman, S. y Palmer, P (2007). Works of art are a good thing to think about: A study of the impact of the Artful Thinking program on students' concepts of thinking. VV.AA., Evaluating the Impact of Arts and Cultural Education, (pp. 89-101). Paris: Centre Pompidou.

VV.AA. (2017). ArtStarts Annual Report. Recuperado de https://artstarts.com/assets/

Yelo, J. J. (2012). [Educa Thyssen] (6-IX-01). Paisajes sonoros. Escuchando la pintura [Archivo de vídeo]. Recuperado de https://www.youtube.com/

Yelo, J. J. (2017). Composición en el aula de paisajes sonoros urbanos imaginados: Las Ciudades Invisibles de Italo Calvino. En J. L. Carles y A. Núñez (eds.), Espacios Sonoros y Audiovisuales 2016: La ciudad como lugar de Experimentación, Creación e Investigación Plurisensorial (pp. 82-88). Madrid: autoedición.

JUAN JESÚS YELO CANO. THE CONTENT OF THIS ARTICLE IS THE SOLE RESPONSIBILITY OF THE AUTHORS. THE REVISTA ELECTRÓNICA DE LEEME AND UNIVERSITAT DE VALĖNCIA ARE NOT LIABLE FOR ANY LEGAL ACTIONS THAT MAY ARISE INVOLVING THE ARTICLE'S CONTENT. REVISTA ELECTRÓNICA DE LEEME -LISTA ELECTRÓNICA EUROPEA DE MÚSICA EN LA EDUCACIÓN-. HTTP://OJS.UV.ES/INDEX.PHP/LEEME/INDEX ISSN: 1575-9563 EDITORES: UNIVERSIDAD DE VALENCIA Y JESÚS TEJADA GIMÉNEZ. VISIBILIDAD DE ESTA REVISTA: SCOPUS, EMERGING CITATION INDEX (CLARIVATE), EBSCO, CINDOC (CSIC), CITEFACTOR, COPAC, DIALNET, DICE (CSIC), DOAJ, E-REVISTAS (CSIC), EBSCO PREMIER, ERIH+, GALE CENGAGE LEARNING, IN-RECS, IRESIE, LATINDEX, MIAR, OCLC WORLDCAT, RESH, REDIB, RILM CORE JOURNALS, SUDOC, ULRICHS, ESTA REVISTA ESTÁ PUBLICADA CON EL APOYO INSTITUCIONAL DE REDIRIS-CONSEJO SUPERIOR DE INVESTIGACIONES CIENTIFICAS Y ES DE ACCESO LIBRE. CREATIVE COMMONS LICENSE 4.O BY 


\section{Anexo I}

\section{Encuesta de valoración}

1. Si has realizado la tarea en pareja, ¿cómo ha sido el trabajo con tu compañera?

a. Yo tenía la mayoría de ideas

b. Me he dejado llevar por mi compañera

c. Hemos trabajado conjuntamente; las dos hemos aportado ideas y sugerencias

2. ¿Crees que después de realizar este proyecto conoces alguno de los procesos que siguen los artistas para componer sus obras?

a. Sí, creo que entendiendo mejor cómo los artistas crean sus obras

b. No, no creo haber aprendido nada sobre cómo hacen los artistas para crear sus obras

3. ¿Cuál dirías que ha sido el proceso que habéis llevado a cabo en la creación de vuestra obra?

a. Hemos ido improvisando conforme íbamos grabando

b. Primero hemos planificado el trabajo y cuando ya lo teníamos todo claro lo hemos grabado

c. Hemos probado varias cosas y nos hemos quedado con lo que más no ha gustado

4. La tarea de realizar una reinterpretación del texto y la imagen de dos artistas murcianos te ha parecido...

a. Interesante y/o agradable

b. Me ha sido indiferente

c. Aburrida

d. Otro

5. ¿Qué dirías que ha aportado este trabajo a tu formación como persona?

6. ¿Qué opinas de que tu trabajo forme parte de la exposición cuando se haga en otra ciudad?
a. Me parece muy bien
b. Me parece bien
c. Me parece mal
d. Me parece muy mal
e. Me da igual

7. Te parece muy bien/bien/mal/muy mal/me da igual que tu trabajo forme parte de la exposición cuando se haga en otra ciudad porque...

8. Escoge la opción que mejor defina cómo te has sentido al terminar tu trabajo
a. Contenta, he sido capaz de componer una obra con valor artístico
b. Decepcionada, no me ha gustado el resultado
c. No he sentido nada especial, ha sido un trabajo escolar más
d. Cansada, me ha costado mucho trabajo hacerla
e. Otro 